\title{
Dark heterochromia in adult masked lapwings is universal, asymmetrical and possibly slightly sexually dimorphic
}

\author{
Adam P. A. Cardilini ${ }^{1} \cdot$ Daniel Lees $^{2} \cdot$ Dylan Roche $^{1} \cdot$ Peter Dann $^{3} \cdot$ Michael A. Weston $^{2}$ (i)
}

Received: 25 October 2021 / Accepted: 31 December 2021 / Published online: 17 January 2022

(c) The Author(s) 2022

\begin{abstract}
Variations in eye colour in birds are poorly documented. We measured and characterised eyes of 25 birds in the hand $\left(16^{\circ} \times 22.5^{\circ}\right.$ segments per eye), using standardised and scaled images, and examined observations of 1 marked individual through time. We describe universal and extensive dark heterochromia (non-uniform colouration of the eye) in adult masked lapwings Vanellus miles novaehollandiae in our study population. Asymmetrical within-eye heterochromia has not previously been recorded for Charadridae, but in a review of images of eyes in the genus Vanellus, we found $45 \%$ of the 22 species had detectable heterochromia. There was a tendency ( $p=0.051$ after false discovery rate correction) for males to have slightly less black in the upper posterior part of the eye, a difference not distinguishable enough to aid sexing in the hand. We suggest standardised images of captured birds would underpin a more nuanced understanding of the occurrence of subtle heterochromia among birds, which may permit phylogenetic analyses directed at exploring possible evolution or function of such eye pigmentation.
\end{abstract}

Keywords Eyes $\cdot$ Coloration $\cdot$ Pigmentation $\cdot$ Shorebirds

\section{Zusammenfassung}

Dunkle Heterochromie bei adulten Maskenkiebitzen ist universell, asymmetrisch und möglicherweise leicht geschlechtsdimorph

Variationen der Augenfarbe bei Vögeln sind nur unzureichend dokumentiert. Wir vermaßen und charakterisierten Augen von 25 Vögeln in der Hand $\left(16 \times 22,5^{\circ}\right.$ Segmente per Auge), wobei wir standardisierte und skalierte Abbildungen verwendeten, und untersuchten Beobachtungen eines markierten Individuums im Laufe der Zeit. Wir beschreiben eine universelle und ausgedehnte dunkle Heterochromie (uneinheitliche Färbung des Auges) bei adulten Maskenkiebitzen Vanellus miles novaehollandiae in unserer Studienpopulation. Asymmetrische Heterochromie innerhalb des Auges wurde bei Charadridae bisher noch nicht festgestellt. Jedoch fanden wir bei einer Überprüfung von Augenabbildungen der Gattung Vanelllus, dass bei $45 \%$ von 22 Arten eine Heterochromie nachweisbar war. Bei den männlichen Maskenkiebitzen gab eine Tendenz ( $\mathrm{p}=0,051$ nach Korrektur anhand der Falscherkennungsrate; engl. False Discovery Rate, FDR) zu etwas weniger

Communicated by I. Moore.

Michael A. Weston

mike.weston@deakin.edu.au

1 Faculty of Science, Engineering and the Built Environment, School of Life and Environmental Sciences, Deakin University, 75 Pigdons Road, Waurn Ponds, Geelong, VIC 3216, Australia

2 Faculty of Science, Engineering and the Built Environment, School of Life and Environmental Sciences, Deakin University, 221 Burwood Highway, Burwood, Geelong, VIC 3125, Australia

3 Research Department, Phillip Island Nature Parks, PO Box 97, Cowes, Phillip Island, VIC 3922, Australia 
schwarz im oberen hinteren Teil des Auges als bei Weibchen. Dieser Unterschied war jedoch nicht deutlich genug, um die Geschlechtsbestimmung in der Hand zu erleichtern. Wir schlagen daher vor, dass standardisierte Abbildungen von gefangenen Vögeln ein differenziertes Verständnis des Auftretens subtiler Heterochromie bei Vögeln ermöglichen würden. Dies könnte phylogenetische Analysen zur Erforschung einer möglichen Evolution oder Funktion einer solchen Augenpigmentierung ermöglichen.

\section{Introduction}

Vision is a prominent form of sensory perception among birds, with great variation in eye form, function and capacity across bird species (Martin 2017). Apart from critical adaptations regarding visual acuity and function, eye colouration can be involved in social signalling (Passarotto et al. 2020), antipredator crypsis (Davidson et al. 2017; Passarotto et al. 2020) and possibly reflect individual quality, condition (Wails et al. 2018) or disease status of individuals (Goodwin 2018). Black or dark brown colouration of the eye of birds (due to both pigmentation and other factors), in the form of areas, flecks or spots, has also been described (Yoo et al. 2017). Such heterochromia (differences in the colouration of different parts of the same anatomical structure, which are usually alike in colour) is often asymmetrical around the iris, and in at least one case can be asymmetrical between eyes (i.e. Common Cuckoo Cuculus canorus) (GutiérrezExpósito 2019; Yoo et al. 2017). Within-eye asymmetrical heterochromia has been recorded from only $5.4 \%$ of the world's bird species, and evidently can vary within species with body mass, age, and occasionally, with sex (GutiérrezExpósito 2019; Yoo et al. 2017). Any functional significance of heterochromia is unknown, however, it may be related to the regulation of light. The presence of dark areas in a pale iris have been hypothesized to serve to minimize the amount of light passing through the iris, and also possibly to reduce glare when a bird is foraging (Gutiérrez-Expósito 2019). Gutiérrez-Expósito (2019) suggested that the dark crescent in the irides of buttonquails may facilitate foraging in these species which move from dark to bright environments when foraging. Yoo et al. (2017) describe the exceptional patterns present in the Common Cuckoo eye, in which the left eye is almost always darker than the right eye, challenging the notion that selection universally drives symmetry among birds. This exceptional pattern might result from betweeneye dominance in birds caused by embryonic positioning within the egg, and selection for between-eye asymmetrical heterochromia to achieve visual laterization (Yoo et al. 2017). This contention, however, requires testing, and the vast majority of birds with heterochromia exhibit betweeneye symmetry in any heterochromia (Yoo et al. 2017).

Eye colouration in wild animals, including birds, is considered understudied (Negro et al. 2017). This is specifically true of heterochromia in the eyes of birds (Gutiérrez-Expósito 2019; Yoo et al. 2017). As part of an urban ecology study of breeding masked lapwings Vanellus miles (Cardilini et al. 2013, 2019), we captured incubating adult birds and discovered apparently universal heterochromia. Heterochromia in birds may not be symmetrical around the pupil, and may form distinct patterns, with a variety of patterns described across Aves (Gutiérrez-Expósito 2019); thus, we quantify the within-eye patterns observed and describe the patterns of heterochromia around the pupil of masked lapwings. Having quantified heterochromia, we examine whether any between-eye-asymmetry exists, as has been described for the Common Cuckoo (Yoo et al. 2017). We then assess whether heterochromia varies between the sexes, as has been proposed for some Oystercatchers (Guzzetti et al. 2008; Rowe and Minton 2016). Finally, we also survey published images for the prevalence of heterochromia in the genus Vanellus, to assess whether such patterns are more widespread, perhaps even typical, of the genus (lapwings). Based on the broad patterns observed in birds (GutiérrezExpósito 2019), and the few studies of heterochromia in shorebirds (Guzzetti et al. 2008; Rowe and Minton 2016), we expect heterochromia will be asymmetrical around the pupil within eyes, symmetrical between eyes, and possibly exhibit subtle variation between the sexes. We also predict that heterochromia is hitherto under-reported across lapwing species, and that some lapwing species other than masked lapwings may exhibit heterochromia.

\section{Methods}

We captured adult, incubating birds of the southern subspecies (V. m. novaehollandiae) using nest traps (see Lees et al. 2021). We took standardised lateral images of their heads, including their left and right eyes. Adult masked lapwings have yellow eyes with black pupils, with dark heterochromia evident on the otherwise yellow irides (Fig. 1). Birds were individually marked with leg flags, and sexed using molecular techniques (see Cardilini et al. 2013). We held birds in a standardised fashion, and each image detailed the individual eye and included a scale in the form of a ruler. Images were taken using a Canon DSLR camera (EOS 600D) with a standard lens (EFS 18-55 mm). Photos were taken of the bird's head in a side-on orientation, using automatic settings and forced flash. We were unable to use a light box.

To quantify eye patterns, we required a comparable set of metrics. We defined the centre of the pupil in each image, as 


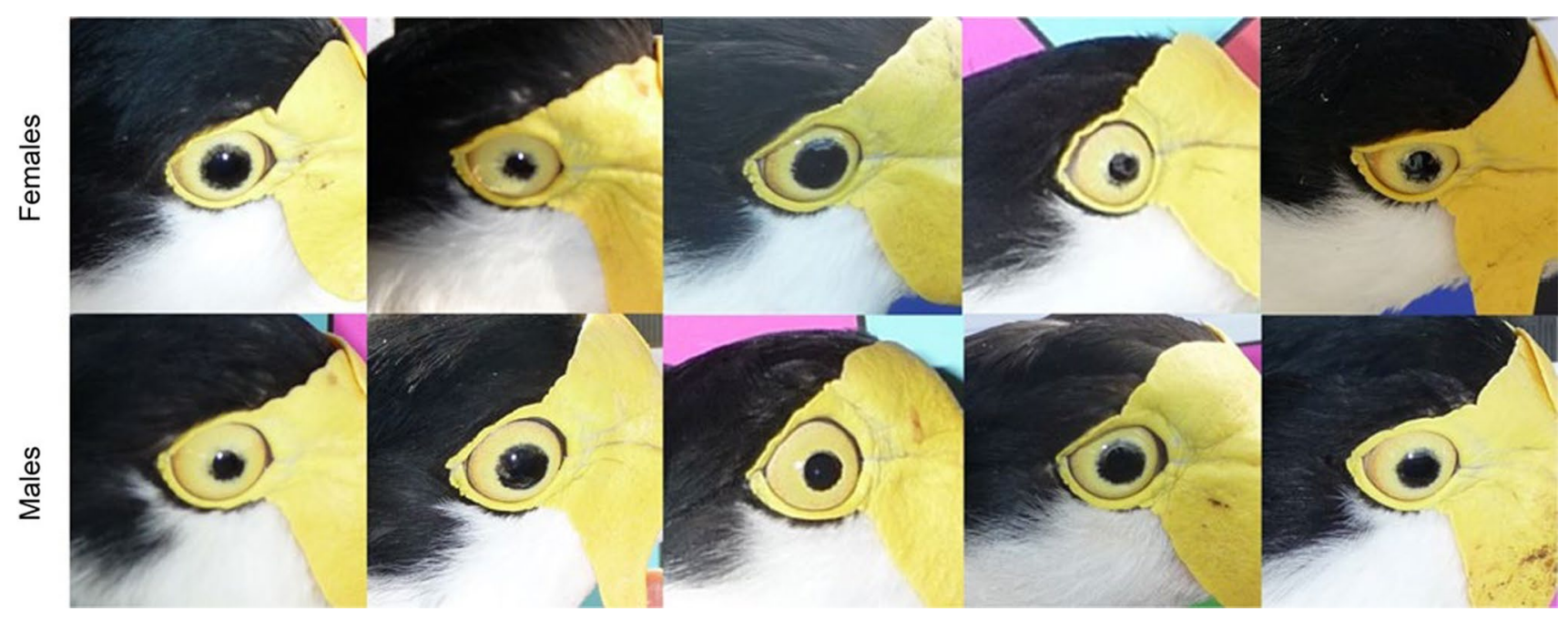

Fig. 1 Selected images of left eyes from a subset of standardised images of masked lapwings. Dark heterochromia is clearly visible on all eyes. Bills are to the right

well as a reference line (the line perpendicular to the mandibular intersection, where the upper and lower mandibles meet). We then intersected a line perpendicular to the reference line, and which passed through the centre of the pupil (Fig. 2). This permitted the reliable orientation of an overlay of $16^{\circ} \times 22.5^{\circ}$ segments per eye (Fig. 2). For each segment, we measured the following: (1) extent of projection (the distance from eye centre to the furthest interface between black and the yellow iris); (2) 'colour' of the zone between the heterochromia at its outer margin (black, 5; dark grey, 4; light grey, 3; faint grey, 2; none, 1); and (3) a 'colour block' scale which measured how uniform any heterochromia was in each segment (none, 1; partial/spotted, 2; full, 3). Distances were scaled to the ruler in the image, and expressed as millimetres. A single investigator (A.P.A.C.) coded all images, and was unaware of the sex of the individual birds being examined.

To ascertain the extent to which heterochromia is present in the genus Vanellus we examined high-resolution photographs from the ebird website (Sullivan et al. 2009; 28 February 2021) of the 21 other (i.e. excluding masked lapwing) extant members of the genus (Gill et al. 2020). We located as many high-resolution images of adults of each species as possible, and where some uncertainty remained regarding the presence of heterochromia, we examined up to a maximum of 50 images. By zooming in on each eye, we carefully inspected each. When a bird had black or grey flecking/ colouration in the iris, that was regarded as heterochromia, regardless of whether or not it was symmetrical around the pupil. For some species, heterochromia was evident in some but not all images; in these cases, we regarded the species
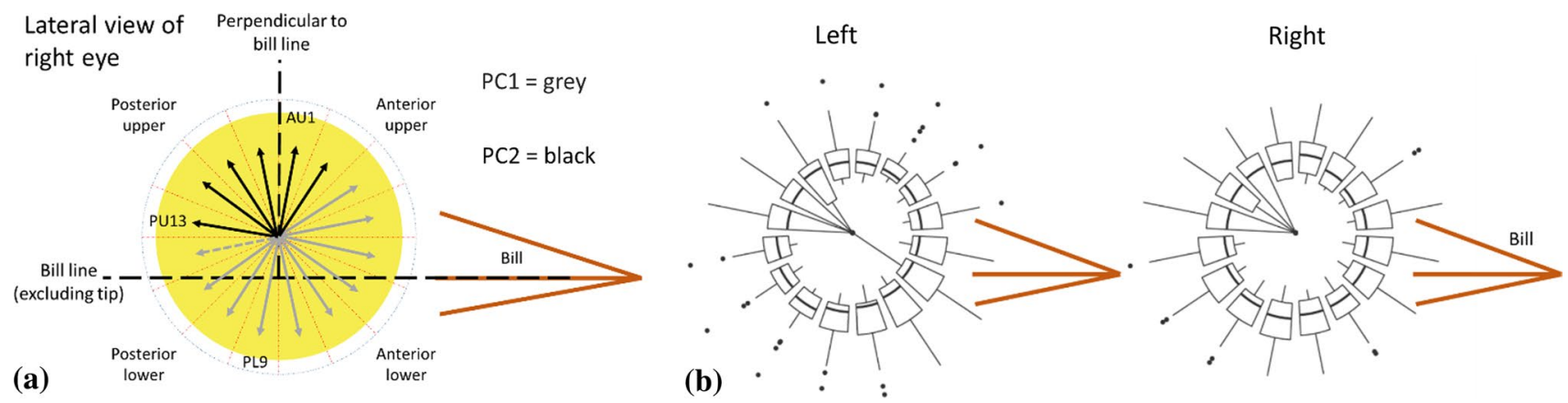

Fig. 2 The segments used to characterise and measure dark heterochromia (a), which are oriented based on the black dashed lines indicated. Segments with grey arrows are associated with longer projections (i.e. segments with factor loadings $>0.5$ ) in the lower, anterior aspect $(\mathrm{PC} 1)$, black arrows with longer projections in the posterior

upper aspect (PC2). The dashed arrow indicates a segment which was loaded positively against both components, but more so for PC1. Box plots of the length of heterochromia in left and right eyes of masked lapwings $\mathbf{b}$ are also shown (bill to the right in each case) 
as exhibiting heterochromia but note that some individuals may not exhibit it.

We used principal components analysis (PCA) to reduce the dimensionality of our continuous data (extent of projection) and to derive a model of our data (implemented in SPSS v. 27). We use rotated component scores as response variables in linear mixed models (LMMs) to test for the effect of sex on the extent of heterochromia, while accounting for the fact that birds have two non-independent eyes (i.e. bird identity was a random effect). Given that the Principal Component (PC) scores were correlated, we adjusted our $p$ values for false discovery rates (to adjust for an inflated type 1 error rate). Patterns in colour and colour blocks were ordinal in nature, and we here apply multivariate statistics which allow the incorporation of a random effect of bird identity (i.e. Permutational Multivariate Analysis of Variance [PERMANOVA]), followed by an exploration of dispersion (using PERMDISP, Permutational Analysis of Multivariate Dispersions) (implemented in PRIMER v. 7). Non-metric Multidimensional Scaling (nMDS) was used to visualise results (Anderson et al. 2008).

\section{Results}

Heterochromia was universal in our sample (100\% of 25 birds) and also widespread in the eyes of the birds in the hand which we examined. Of the 16 segments of 49 eyes (784 segments), some heterochromia was evident in $89.7 \%$ of segments ( 25 birds, of which 24 had both eyes photographed, and 1 bird had 1 eye photographed). The extent of projection averaged $2-4 \mathrm{~mm}$ for all segments. The main parts of the eye which may have no heterochromia were in the upper posterior quadrant (segments, PL 13-15; see Fig. 2). Examples of the heterochromia we observed are provided in Fig. 1.

The extent of projections of each of the 16 segments quantified from birds in the hand were generally highly correlated with 1 another, with some exceptions, but no correlation was lower than 0.377 . To characterise heterochromia across the eye, we conducted PCA with Oblimin rotation (as components were correlated when two factors were extracted, $r=0.564$ ) for each eye measured (Kieser-Meyer-Olkin measure of sampling adequacy, 0.903; Bartlett's test of sphericity, $\left.\chi_{120}^{2}=1448.771, p<0.001\right)$. We interpret two components with eigen values $>1$. Higher PC1 scores (explaining $72.8 \%$ of variation, Cronbach's $\alpha=0.963$ ) were associated with loadings $>0.5$ for segments in the lower anterior, posterior and upper anterior quadrants and PC2 scores (14.4\%; $\alpha=0.977$ ) were associated with higher loadings with upper posterior aspects (Fig. 2). Given that some species exhibit systematic differences in black flecking between left and right eyes (Yoo et al. 2017), we tested whether this was the case for masked lapwings in the hand, but no difference between left and right eyes was evident (Paired $T$ tests of component scores: PC1, $T=-0.406, d f=23, p=0.689$; PC2, $T=0.166, d f=23, p=0.869)$. We ran separate LMMs for each PC score, featuring sex as a fixed factor, and bird identity as a random intercept (Gaussian distribution). For PC 1 (lower anterior, posterior and upper anterior quadrants), there was no effect of $\operatorname{sex}(T=-1.126, p=0.260$; after False Discovery Rate correction), however, for PC2 (upper posterior) sex approached significance $(T=-2.231$, $p=0.051$; after False Discovery Rate correction $[p=0.026$ without correction]) with males having lower scores $(-0.306 \pm 0.235$ [mean \pm SE] $)$ than females $(0.491 \pm 0.288)$. This suggests that females have slightly more upper posterior heterochromia, although we do not consider this diagnostic, as males and females vary greatly in this respect (see Fig. 1).

Median colour and colour block for each eye did not differ between eyes within birds (birds in the hand; Wilcoxon signed-rank tests; colour, $W=-1.180, p=0.238$; colour block, $W=-0.452, p=0.651)$. We had one more right eye than left eyes, so examined median colour and colour block for right eyes across sexes. These did not differ with sex (Mann-Whitney tests; colour, $U=-0.201, p=0.849$; colour block, $U=-0.068, p=0.978)$. To further test for patterns in colour and colour blocks in the heterochromia between sexes within eyes, we generated Euclidean resemblance matrices, and performed separate PERMANOVAs, with sex as a fixed effect and bird identity as a random effect. There was no effect of sex on colour (birds in the hand; Psuedo $\left.F_{1,24}=0.854, p=0.516\right)$. PERMDISP revealed a difference in dispersion between sexes $\left(F_{1,47}=7.8038, p=0.016\right)$, a visual inspection of the nMDS suggests this is driven by male eyes exhibiting higher colour scores in heterochromia than females in parts of the anterior lower and upper quadrants (Fig. 3). For colour block (another ordinal scale, and a model of the same structure as above), sex approached significance (Psuedo $F_{1,24}=1.9276, p=0.077$ ), despite substantial overlap between the sexes on the nMDS, and the sexes did not differ in dispersion $\left(F_{1,47}=3.41, p=0.107\right)$.

For one bird we captured, we can describe change in heterochromia over time, because we have photos of the bird's eye taken after the initial standardised photograph (Supplementary Fig. 1). Heterochromia in the lower eye in this bird apparently varied over time - the later picture showed darker heterochromia in the lower eye. In the upper eye, heterochromia started out faint and spotted, over time the upper eye developed darker and more consistent heterochromia.

Finally, to establish the prevalence of heterochromia among lapwings, we reviewed 436 photographs from the internet $(20.4 \pm 4.5$ [SE] per species; $4-50)$ and found clear evidence of heterochromia in 9 of the 21 other species 
Fig. 3 Non-metric multidimensional scaling (nMDS) plot of colour at the edge of heterochromia in the eyes of male and female masked lapwings. Males occupy part of the multivariate space not occupied by females (arrow), that associated with greater colour with anterior upper and lower segments of the eye (vectors with $r \geq 0.8$ indicated; see Fig. 2)

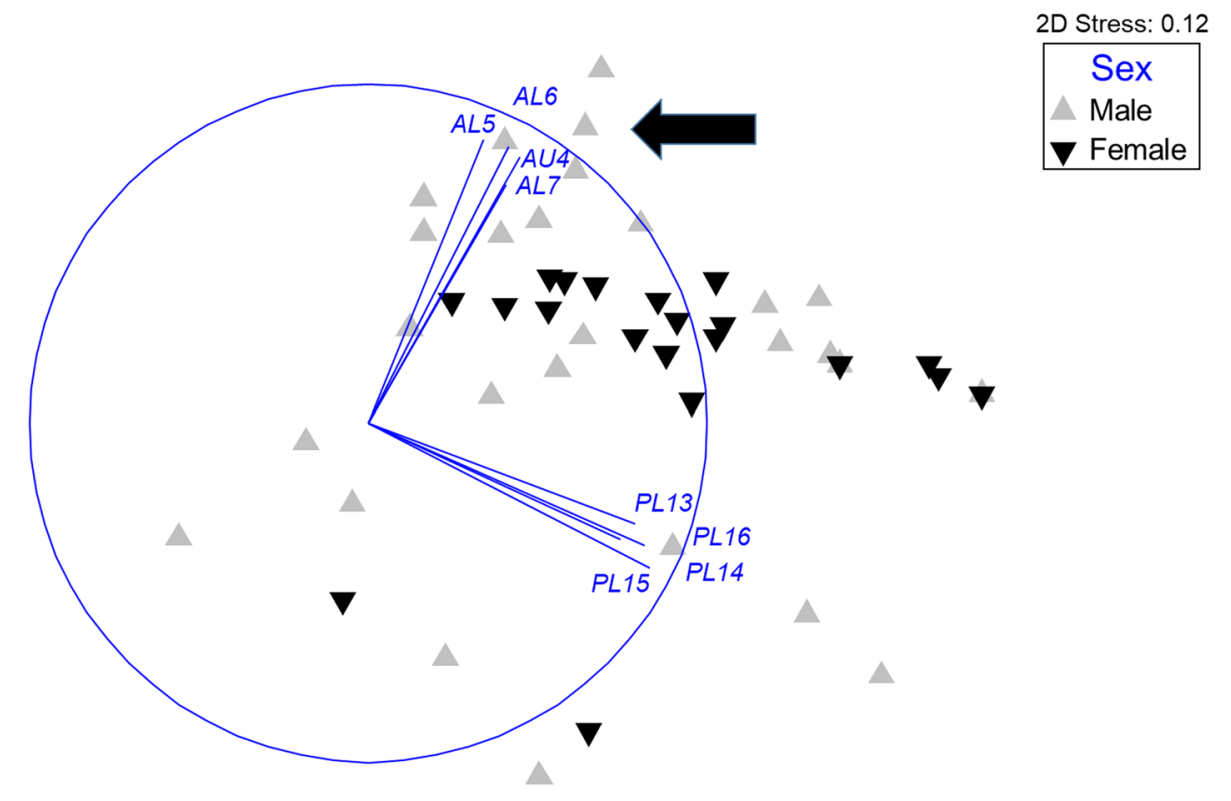

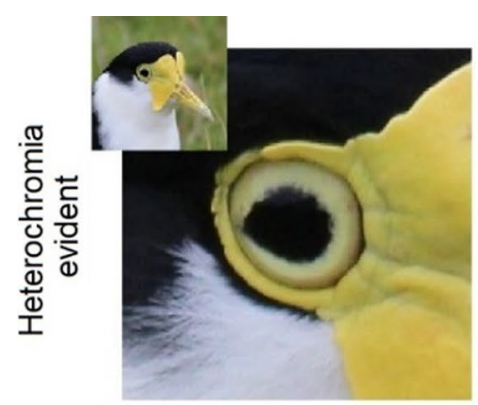

Vanellus miles

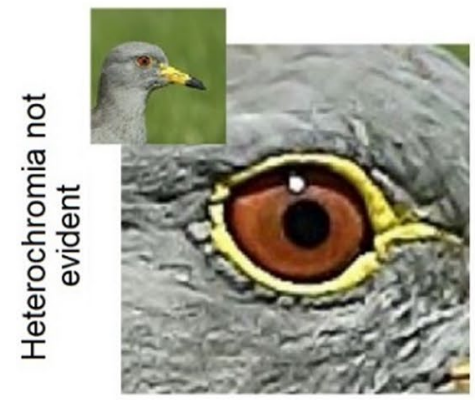

Vanellus cinereus

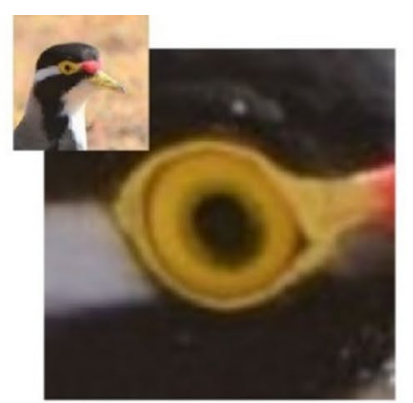

Vanellus tricolor

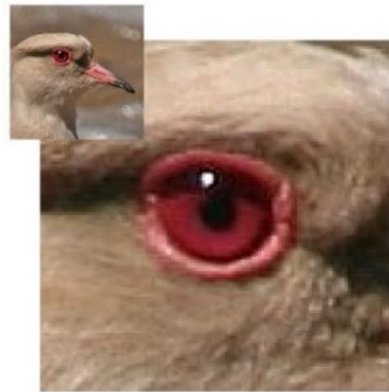

Vanellus resplendens

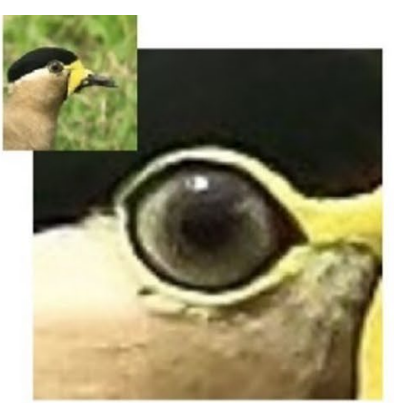

Vanellus malabaricus

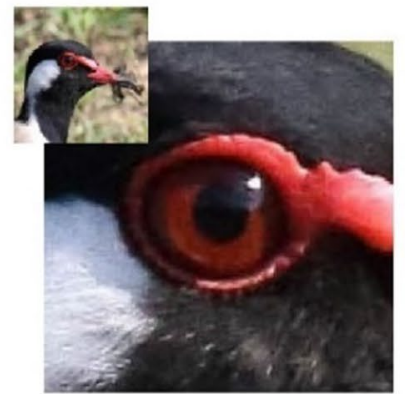

Vanellus indicus

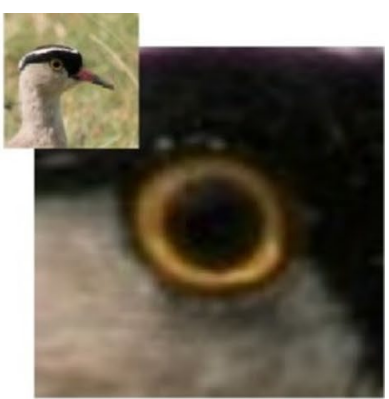

Vanellus coronatus

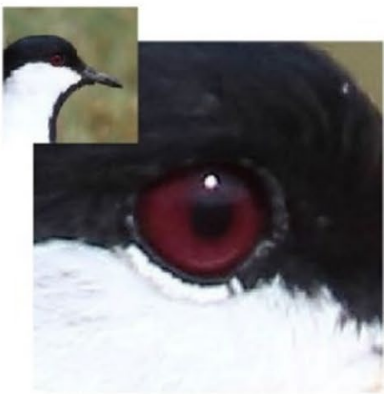

Vanellus spinosus

Fig. 4 Examples of images of lapwing eyes used to assess the occurrence of heterochromia in the genus Vanellus. Photos were generously provided by: Daniel Lees, Brian O’Leary, Delip K. Das, Roz Jessop, Peter Johnston and Humphrey P. Sitters

(overall, including the masked lapwing, 45\%; Fig. 4). Species displaying heterochromia are: V. tectus, V. malabaricus, V. lugubris, V. melanopterus, V. coronatus, V. senegallus, V. superciliosus, V. leucurus, V. tricolor and V. miles. It is difficult to assess the presence of heterochromia when the iris is dark, and we also note at least some variation in the presence or detectability of heterochromia between individuals of two species (V. malabaricus and V. superciliosus), so we acknowledge that we may underestimate the occurrence of heterochromia in the genus Vanellus. 


\section{Discussion}

All masked lapwings we examined in the hand had heterochromia. The existence of two principal components in extent of projection suggests within-eye-asymmetrical heterochromia i.e. although correlated, black/grey colouration in the irides of lapwings appears to differ between the lower anterior and upper posterior aspects. Such asymmetry is, however, subtle (Fig. 1). Asymmetrical-within-eye heterochromia has been described by Gutiérrez-Expósito (2019) in only 5.4\% of the world's bird species belonging to nine families, which did not include lapwings (Charadridae, which includes the formerly recognised Vanellidae). Based on a review of images from the internet, we report heterochromia in $45 \%$ of species in the genus Vanellus. We may have underestimated prevalence, where irides were dark (see Gutiérrez-Expósito 2019) or under the possibility that within-species variation occurs (which we also report for at least some species) and/or we failed to sample enough individuals. In terms of shorebirds, dark within-eye asymmetrical heterochromia has previously been reported only in Hematopodidae and Pedionomidae (Gutiérrez-Expósito 2019). We here report it also for Charadridae, genus Vanellus. While most shorebirds forage in open areas, many forage by day and night, so the idea that heterochromia may aid vision in varying light conditions as in buttonquails (Gutiérrez-Expósito 2019), may also apply to shorebirds.

Gutiérrez-Expósito (2019) described four patterns of heterochromia (from 85 species with heterochromia). The widespread nature of darker colouration we describe around the pupil of masked lapwings evidently exceeds the extent of colour described in the aforementioned four patterns. The pioneering work of Gutiérrez-Expósito (2019) in mapping the occurrence of heterochromia across Aves could be augmented by standardised images of captured birds, and quantification of patterns. We suggest standardising light levels, and having high-resolution images of birds in the hand (as opposed to images of wild living birds) may lead to the discovery of additional occurrences of heterochromia (David et al. 2013; Polakowski et al. 2020). We have also offered a template for quantifying heterochromia, and describe several metrics which may prove useful.

While studies are few, we are not the first study to describe possible sex-based differences in heterochromia among shorebirds; Guzzetti et al. (2008) found a dark crescent in the eyes of all adult female Black Oystercatchers (Haematopus bachmani) but only in $65 \%$ of the adult males. Australian Pied Oystercatchers H. longirostris, whose sex was inferred by bill measurements, were more likely to be male if they had "fully" pigmented irides (Rowe and Minton 2016). We describe the masked lapwing as a third shorebird species in which adult males might have less black in the iris than females, but in which all birds have some black around the iris. The functional significance, if any, of asymmetrical heterochromia is unknown but possibly related to regulation of light (Gutiérrez-Expósito 2019). Thus, differences between sexes might reflect sex specialisations in foraging, habitat use, or predator detection perhaps related to sex roles in parental care. Masked lapwing partners generally remain together in the same open habitats where they forage (Cardilini et al. 2013), and while no specific studies of foraging are available, diet in the study area does not appear to differ between the sexes (or across seasons; Supplementary Fig. 2). Masked lapwings also share incubation by day and night thereby requiring similar predator detection capacities (Cardilini et al. 2015). This suggests these explanations are unlikely to apply in this case. Subtle, sex-specific foraging specialisations remain unstudied, and may explain these differences.

Some types of heterochromia can vary over time within individuals (Guzzetti et al. 2008), and we show a change in pattern of black heterochromia in an individual adult masked lapwing photographed over time. Masked lapwing chicks have brown irides, but we did not have standardised images of known individuals to document colour change with growth. Eye colour changes with age in many species (Polakowski et al. 2020), and older Australian Pied Oystercatchers are more likely to have fully pigmented irides (Rowe and Minton 2016), with pigmentation evidently increasing with age post-sexual maturity. All the lapwings we captured were breeding adults when first caught (Lees et al. 2021), so changes reflect those which occur post-adulthood. It could be that age-related factors mediating heterochromia continue beyond first breeding, or that heterochromia varies, in relation to internal or external factors (e.g. age and sex).

\section{Conclusions}

Standardised images offer a key resource for studies of colouration in the avian eye. Their promise as a tool to facilitate sexing and ageing (Guzzetti et al. 2008; Rowe and Minton 2016), suggests that it would be ideal if they became part of the standard data collected by bird banders.

Supplementary Information The online version contains supplementary material available at https://doi.org/10.1007/s10336-021-01959-w.

Acknowledgements The Australian Bird and Bat Banding Scheme (ABBBS) kindly provided the metal rings. Some editorial work occurred at the Beach Ecology and Conservation Hub (BEACH; Venus Bay).

Author contributions Conceptualization, AC and MAW; methodology, AC and MAW; formal analysis, AC, DR, DTL and MAW; 
investigation, all; resources, PD and MAW; data curation, AC, DR and DTL; writing — original draft preparation, MAW; writing—review and editing, all; supervision, MAW and PD; project administration, MAW. All authors have read and agreed to the published version of the manuscript.

Funding Open Access funding enabled and organized by CAUL and its Member Institutions.

Data availability statement Data are available upon request to the corresponding author.

\section{Declarations}

Conflict of interest The authors declare no conflict of interest.

Approvals Work was conducted under Deakin University Animal Welfare Committee Permits B02-2012, State Government Permits 10006205, and Australian Bird and Bat Banding Scheme (ABBBS) Authorities 1763, 3271 and 3033.

Open Access This article is licensed under a Creative Commons Attribution 4.0 International License, which permits use, sharing, adaptation, distribution and reproduction in any medium or format, as long as you give appropriate credit to the original author(s) and the source, provide a link to the Creative Commons licence, and indicate if changes were made. The images or other third party material in this article are included in the article's Creative Commons licence, unless indicated otherwise in a credit line to the material. If material is not included in the article's Creative Commons licence and your intended use is not permitted by statutory regulation or exceeds the permitted use, you will need to obtain permission directly from the copyright holder. To view a copy of this licence, visit http://creativecommons.org/licenses/by/4.0/.

\section{References}

Anderson MJ, Gorley RN, Clarke KR (2008) PERMANOVA+ for PRIMER. Primer-e Ltd, Devon

Cardilini AP, Weston MA, Nimmo DG, Dann P, Sherman CD (2013) Surviving in sprawling suburbs: suburban environments represent high quality breeding habitat for a widespread shorebird. Landsc Urban Plann 115:72-80

Cardilini AP, Weston MA, Dann P, Sherman CD (2015) Sharing the load: role equity in the incubation of a monomorphic shorebird, the masked lapwing (Vanellus miles). Wilson J Orn 127:730-733

Cardilini AP, Lees D, Dann P, Weston MA (2019) Higher rates of non-breeding territory occupancy of urban compared with rural masked lapwings Vanellus miles on Phillip Island, Victoria. Vic Nat 136:96-100

David S, Muñoz JM, David O, Estrada-Flórez MC, Cuervo AM (2013) Iris coloration and a case of temporary heterochromia in the Redbellied Grackle (Hypopyrrhus pyrohypogaster). Ornitol Colomb 13:83-88

Davidson GL, Thornton A, Clayton NS (2017) Evolution of iris colour in relation to cavity nesting and parental care in passerine birds. Biol Lett 13:20160783

Gill, F, Donsker, D, Rasmussen, P (2020) IOC World Bird List (v102). https://doi.org/10.14344/IOCML102

Goodwin W (2018) Is there a link between eye colour and haemoparasite infection in the Southern Boobook Ninox boobook in the Perth Metropolitan Area? Aust Field Orn 35:113-116

Gutiérrez-Expósito C (2019) Asymmetric Iris Heterochromia in Birds: the Dark Crescent of Buttonquails. J Ornithol 160:281-285

Guzzetti BM, Talbot SL, Tessler DF, Gill VA, Murphy EC (2008) Secrets in the Eyes of Black Oystercatchers: a New Sexing Technique. J Field Ornithol 79:215-223

Lees D, Cardilini AP, Sherman CD, Dann P, Weston MA (2021) Adult capture on the nest does not affect hatching success of masked lapwing (Vanellus miles) eggs on a fox-free island. Wildl Res 48:361-365

Martin GR (2017) The sensory ecology of birds. Oxford University Press, Oxford

Negro JJ, Blázquez MC, Galván I (2017) Intraspecific eye color variability in birds and mammals: a recent evolutionary event exclusive to humans and domestic animals. Front Zool 14:1-6

Passarotto A, Cruz-Miralles Á, Avilés JM (2020) Iris yellowness relates to age and individual quality in two owl species. J Raptor Res 54:233-244

Polakowski M, Stępniewski K, Śliwa-Dominiak J, Remisiewicz M (2020) Age-dependent differences in iris colouration of passerines during autumn migration in Central Europe. PeerJ 8:e9188

Rowe M, Minton C (2016) Pied Oystercatchers-are observations of their irides useful in sex determination in the field? VWSG Bull 39:62-64

Sullivan BL, Wood CL, Iliff MJ, Bonney RE, Fink D, Kelling S (2009) eBird: a citizen-based bird observation network in the biological sciences. Biol Cons 14:2282-2292

Wails CN, Oswald SA, Arnold JM, Weidensaul S (2018) The best dressed are less stressed: associations between colouration and body condition in a North American owl. Bird Study 65:505-515

Yoo HN, Lee JW, Yoo JC (2017) Asymmetry of eye color in the Common Cuckoo. Sci Rep 7:1-9

Publisher's Note Springer Nature remains neutral with regard to jurisdictional claims in published maps and institutional affiliations. 